\title{
Covariograms of convex bodies in the plane: A remark on Nagel's theorem
}

\author{
Daniel Neuenschwander
}

Daniel Neuenschwander completed his habilitation at the University of Bern in 1996. He taught at the Universities of Dortmund and Nancy I. Presently, he holds a part-time professorship for insurance mathematics and finance at the University of Lausanne, teaches the same subjects at the University of Bern, and holds a position at the Federal Section of Cryptology in the Department of Defense. His main research interests lie in probability theory (especially on algebraic structures) and its applications in insurance, finance, and cryptology.

\section{Introduction}

Let $K$ be a convex compact subset of the plane and denote by $\lambda$ the Lebesgue measure in the plane. Then we call the family of all areas $\{\lambda(K \cap(K+v))\}_{v \in \mathbb{R}^{2}}$ the covariogram of $K$. It has been shown by Nagel ([5]) (see also Schwab [6] and Bianchi [2]) that if $K$ is a convex polygon, then $K$ is (up to shift and point-reflection) uniquely determined by its covariogram. Bianchi et al. ([4]) (see also Bianchi [3]) have generalized Nagel's theorem to the case where $K$ is a compact convex body in the plane with piecewise $C_{+}^{2}$-boundary (i.e. $C^{2}$ with strictly positive curvature). Of course, the conjecture would be that the assertion is true for all compact convex bodies in any dimension. But in dimensions $d \geq 4$ there are counterexamples. Even in the plane, for non-smooth boundaries, one can not work with curvatures and an approximation argument is not available up to now. Some special cases in higher dimensions have been treated by Schwab ([6]).

Ein allgemeiner geometrischer Problemkreis ist die Frage, welche Angaben ausreichen, um eine geometrische Figur eindeutig zu charakterisieren (z.B. Sehnenlängen, etc.). Nagel hat 1993 gezeigt, dass ein konvexes Polygon in der Ebene (bis auf Verschiebung und Punktspiegelung) eindeutig bestimmt ist durch die Flächen der Durchschnittsmengen der Originalfigur mit all ihren Translaten (das sog. Kovariogramm). Später hat Bianchi diesen Satz verallgemeinert auf alle konvexen Körper in der Ebene mit im wesentlichen stückweise glattem Rand. In dieser Notiz geben wir einen einfachen Beweis für die Tatsache, dass bei punktsymmetrischen konvexen Körpern in der Ebene mit stückweise glattem Rand die Kenntnis des Kovariogramms in einer Umgebung des Randes seines Trägers ausreicht, um den Körper (bis auf Translation) eindeutig zu rekonstruieren. 
The aim of this note is to provide a simple proof of a sharpening of Nagel's theorem in case of point-symmetric compact convex bodies $K$ in the plane with piecewise $C^{2}$ boundaries: It will be shown that in this situation it is sufficient for the unicity of $K$ (up to a shift) to know the covariogram in a neighborhood of the boundary of its support. It would be interesting, even for the plane, what information about not necessarily point-symmetric convex bodies (or even polygons) is contained in the behavior of the covariogram in a neighborhood of the boundary of its support. In our opinion, this would be a challenging subject of further research.

A paper of related interest is Adler, Pyke ([1]).

Bianchi et al. ([4]) used parabolic approximation and Fourier transform in their proofs.

\section{The result and its proof}

Let $\lambda$ be Lebesgue measure in the plane. For a compact convex subset $K$ of the plane and $v \in \mathbb{R}^{2}$, let

$$
C(K, v):=K \cap(K+v) .
$$

Then we will call the function $c(K): \mathbb{R}^{2} \rightarrow[0, \infty[$ given by

$$
c(K)(v):=\lambda(C(K, v))
$$

the covariogram of $K$ (cf. Nagel [5]).

Theorem 1 Let $K_{1}, K_{2}$ be point-symmetric compact convex bodies in the plane whose boundaries are piecewise two times continuously differentiable. Then, if the supports of the covariograms $c\left(K_{1}\right)$ and $c\left(K_{2}\right)$ have the same boundary $D$ and if $c\left(K_{1}\right)$ and $c\left(K_{2}\right)$ coincide in a neighborhood of $D$, it follows that $K_{2}=K_{1}+w$ for some $w \in \mathbb{R}^{2}$.

The idea of the proof will be to reconstruct the curvature at any smooth point of the boundary of the body and the angle at any vertex. Among other tools, we will use the following lemma on segments of circles:

Lemma 1 Let $F_{r}(\varepsilon)$ be the area of the segment of height $\varepsilon$ of a circle with radius $r$. Then we have:

$$
\lim _{\varepsilon \rightarrow 0} \frac{F_{r}(\varepsilon)}{\varepsilon^{3 / 2}}=\frac{\sqrt{8 r}}{3} .
$$

Proof. Let $\varphi$ be the angle subtended by the arc of the above-mentioned segment at the center of the circle (cf. Fig. 1).

W.l.o.g. we may assume $r=1$. Then we get

$$
F_{1}(\varepsilon)=\frac{1}{2}(\varphi-\sin (\varphi))
$$

On the other hand, since

$$
\cos (\varphi / 2)+\varepsilon=1
$$

and thus

$$
\tan (\varphi / 2)=\frac{\sqrt{2 \varepsilon-\varepsilon^{2}}}{1-\varepsilon},
$$




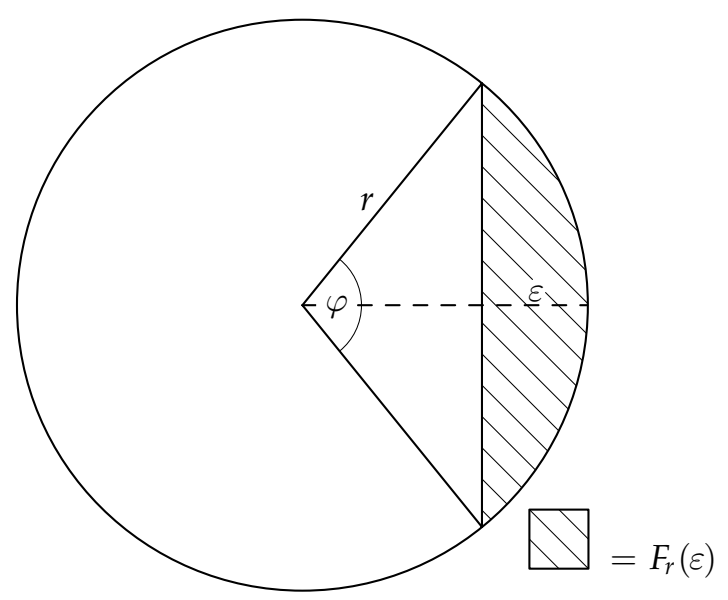

Fig. 1

we get from (1), (2), and (3)

$$
F_{1}(\varepsilon)=\frac{1}{2}\left(2 \arctan \left(\frac{\sqrt{2 \varepsilon-\varepsilon^{2}}}{1-\varepsilon}\right)-2 \sqrt{2 \varepsilon-\varepsilon^{2}} \cdot(1-\varepsilon)\right) \sim \frac{\sqrt{8}}{3} \varepsilon^{3 / 2} \quad(\varepsilon \rightarrow 0)
$$

by developing the arctan function in a MacLaurin series up to terms of third order and using de l'Hospital's rule.

Now we may come to the proof of Theorem 1 .

Proof. W.l.o.g. we may assume that the origin is the center of symmetry of both $K_{1}$ and $K_{2}$. Then we will have to show that under the assumptions of the theorem, it follows that $K_{1}=K_{2}$. Let $\varepsilon>0$ always be small enough. Put $K=K_{1}$ and let $P=\left(x_{0}, y_{0}\right)$ be any point on the boundary $D=D(K)$ of $K$ at which $D$ has positive finite curvature $\sigma=\sigma(P)$. W.1.o.g. we may assume that $y_{0}=\min \{y \in \mathbb{R}:(x, y) \in D$ for some $x \in \mathbb{R}\}$. Put $v_{\varepsilon}:=\left(2 x_{0}, 2 y_{0}-2 \varepsilon\right)$ and $f(P, \varepsilon):=\lambda\left(K \cap\left(K+v_{\varepsilon}\right)\right)$. Let $A(P, \varepsilon)$ denote the segment of height $\varepsilon$ of the circle of curvature of $D$ at the point $P$ and $F(P, \varepsilon):=\lambda(A(P, \varepsilon))$ its area (cf. Fig. 2).

Define

$$
\begin{aligned}
& J:=\left\{x \in \mathbb{R}:(x, y) \in K \cap\left(K+v_{\varepsilon}\right) \cap A(P, \varepsilon) \text { for some } y \in \mathbb{R}\right\} \quad \text { and } \\
& I:=\left\{x \in \mathbb{R}:(x, y) \in\left(K \cap\left(K+v_{\varepsilon}\right)\right) \cup A(P, \varepsilon) \text { for some } y \in \mathbb{R}\right\} .
\end{aligned}
$$

Then one sees that $f(P, \varepsilon)=2 F(P, \varepsilon)+O\left(\varepsilon^{2}\right)(\varepsilon \rightarrow 0)$ (observe that the difference in the vertical coordinates between points $Q=(x, y)$ on $D$ and their counterparts $Q^{\prime}=\left(x, y^{\prime}\right)$ on the semi-circle of curvature is $O\left(\varepsilon^{2}\right)$ uniformly for all $x \in J$ by definition of the radius of curvature; the same then follows automatically uniformly for all $x \in I$ ). Lemma 1 yields

$$
\lim _{\varepsilon \rightarrow 0} \frac{F(P, \varepsilon)}{\varepsilon^{3 / 2}}=\frac{\sqrt{8}}{3 \sqrt{\sigma}} .
$$




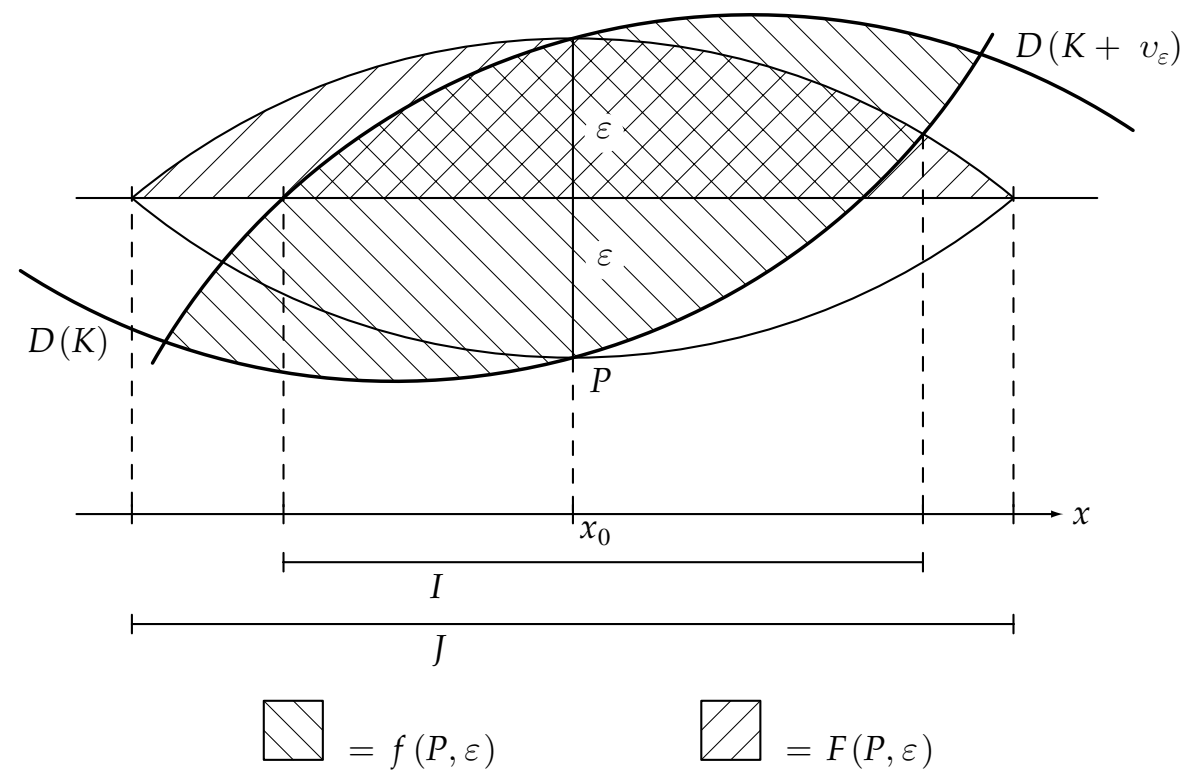

Fig. 2

So, since by assumption the function $f(P, \varepsilon)$ is known, it is possible to reconstruct the curvature $\sigma(P)$ wherever it is positive and finite. On the other hand, corners of $D$ can be "rounded off" with a uniform (for $x$ in a neighborhood of $x_{0}$ ) error of $O\left(\varepsilon^{2}\right)(\varepsilon \rightarrow 0$ ), so also the fact if $\sigma(P)=\infty$ or not can be retrieved by the same method. By continuity (at isolated points $P$ of $D$ with $\sigma(P)=0$ ) or (for points $P$ in straight line segments of $D$ ) by a similar approximation argument as before (here, instead of "rounding off" corners, we have to consider little "bendings" of straight line segments of $D$ ) we also can find out if $\sigma=0$ or not. Hence the curvature $\sigma \in[0, \infty]$ at any point of $D$ can be retrieved by the data $f(P, \varepsilon)$ for all $P \in D$ and $\varepsilon>0$ small enough. After having determined the curvature at all points one can, by a similar consideration of the function $f(P, \varepsilon)$ as above at corners $P=\left(x_{0}, y_{0}\right)$ of $D$ (i.e. points $P \in D$ with $\sigma(P)=\infty$ ), reconstruct the angles at the corners as follows: W.l.o.g. we may assume that the left-hand side derivative is minus the right-hand side derivative of $D$ at $x_{0}$; then (similarly as before) the areas of the isosceles triangles of height $\varepsilon$ formed by a horizontal base and the right- and left-hand side tangent lines of $D$ at $P$ are known up to an error of $O\left(\varepsilon^{2}\right)$ (uniformly for $x$ in a neighborhood of $x_{0}$ ) as $\varepsilon \rightarrow 0$; with that, one is able to determine the angle of $D$ at $P$. It follows that all curvatures $\sigma \in[0, \infty]$ and all angles of the boundary $D$ are uniquely determined by the family of functions $\mathscr{F}=\{f(P, \varepsilon)\}_{P \in D}$ and thus the boundaries of $K_{1}$ and $K_{2}$ coincide. Since (by compactness) $\mathscr{F}$ only depends on the behavior of the covariogram of $K$ in a neighborhood of the boundary of its support, we have finished the proof.

\section{Acknowledgements}

The author wishes to thank Profs. H. Carnal, P. Mani-Levitska and G. Bianchi and Dipl. Math. K. Rufibach for helpful discussions. 


\section{References}

[1] Adler, R.J., Pyke, R.: Scanning Brownian processes. Adv. Appl. Prob. (SGSA) 29 (1997), 295-326.

[2] Bianchi, G.: Determining convex polygons from their covariograms. Preprint 2001.

[3] Bianchi, G.: Determining convex bodies with piecewise $C^{2}$-boundary from their covariograms. Preprint 2001.

[4] Bianchi, G., Segala, F., Volcic, A.: The solution of the covariogram problem for plane $C_{+}^{2}$-bodies. Quad. Matematici Dip. Sci. Mat. Universita di Trieste 485 (2000).

[5] Nagel, W.: Orientation-dependent chord length distributions characterize convex polygons. J. Appl. Prob. 30 (1993), 730-736.

[6] Schwab, S.: Das Problem der Phasenrekonstruktion für Gleichverteilungen auf konvexen Körpern. Diplomarbeit Universität Bern 1998, Phil.-Nat. Fakultät.

Daniel Neuenschwander

University of Lausanne

Institut de Sciences actuarielles

CH-1015 Lausanne

University of Bern

Institut für mathematische Statistik und Versicherungslehre

Sidlerstrasse 5

CH-3012 Bern

and

Federal Section of Cryptology

Department of Defense

CH-3003 Bern, Switzerland

e-mail: daniel .neuenschwander@bluewin.ch 